\title{
The Effect of Using Directional Antennas on Adjacent Channel Interference in 802.11a: Modeling and Experience With an Outdoors Testbed
}

\author{
Vangelis Angelakis ${ }^{+}$, Nikos Kossifidis, Stefanos Papadakis, Vasilios Siris, Apostolos Traganitis \\ Telecommunications and Networks Laboratory, \\ Institute of Computer Science, FORTH. \\ 100 N. Plastira, Vassilika Vouton, 71110 Heraklion, Greece.
}

\begin{abstract}
We present an outdoors 802.11a testbed based on offthe-shelf components that we used for multi-radio node experimentation. This is the first such testbed, to our knowledge, equipped with directional antennas. With it we conducted a thorough and systematic set of measurements, in medium range outdoors links, to examine how the physical separation of the antennas, the output power of the interfaces, and the channel separation of the links affect throughput. For the setup of the testbed and to estimate the induced ACI we utilized a theoretical model, already verified on an in-lab wireless testbed emulator [10]. We discuss how the model needs to adapt and propose to create a tool for the design of large scale multi-radio wireless networks.
\end{abstract}

Keywords-ACI, Multi-radio, 802.11a, Antenna, Testbed.

\section{INTRODUCTION}

\section{A. Multi-radio networking with 802.11 and experimenting} with testbed platforms

In mesh networking a backbone access node, in contrast to a typical infrastructure Wireless LAN scenario, is no longer required to have its own wired connection to the backbone wired network, but rather rely on other infrastructure mesh nodes to forward its traffic to the wired backbone [1,2]. The key benefit of mesh networking is the fast and inexpensive range extension of the wired network infrastructure. The major technological difference from the typical infrastructure wireless LAN is the shift from single-hop wireless networking to multihop wireless networking. This leads to many possible design choices for the architecture of a wireless mesh node.

Well known capacity issues from the ad-hoc networking area [3] and inexpensive off-the shelf wireless devices of the IEEE 802.11 lettersoup have made the multi-radio design of mesh nodes the most appealing one both for research and production. In such multi-radio designs some of the interfaces form wireless point-to-point links with neighboring nodes for backbone connectivity and some for client access. Each of the wireless interfaces of such a node can be connected to an antenna selected from wide range of characteristics, depending on the overall design of the network.

Analytical and simulation-based approaches for the evaluation of such systems have been found to be limited, due

This work has been supported by the General Secretariat for Research and Technology, Greece, through the projects 05-AKMON-80 \& 05-NON-EU-39 ${ }^{+}$Corresponding author: Vangelis Angelakis: angelak@ics.forth.gr. to coarse simplifications in the cross layer modeling and the widely variant wireless channel.

Testbed platforms and experimental measurements in the past few years are very often devised to provide more realistic performance evaluation of different solutions, taking into account real hardware and opening new areas for hypotheses testing and better understanding of systems closer to production.

\section{B. Previous Work}

In [6] the authors perform one of the earliest studies of the various issues involved in experimenting with a multi-radio wireless testbed. Using 802.11b off-the-shelf Prism 2.5-based radio interfaces for their platform they investigated the different choices one may face in the design of a multi-radio mesh node and reported that simultaneous activation of multiple radios on the same node leads to degradation in performance due to: i) board crosstalk, ii) radiation leakage, and iii) inadequate separation between the antennas. Reproducing their Prism 2.5 single-board multi-radio implementation we attributed most of the reported degradation in throughput to a problematic implementation of the monitoring mode on the HostAP Linux driver for WLAN Prism 2.5-based interfaces which will lead to CPU load of nearly $100 \%$ on the machine that hosts multiple monitoring interfaces. Not using the monitoring mode simply alleviated the reported board crosstalk issue, leaving Adjacent Channel Interference (ACI) as the only source of problem, once the antennas were sufficiently separated.

In [7] the authors move to Atheros based 802.11a interfaces and perform testbed experiments to quantify the effect of ACI on a dual-radio multihop network. Their work includes both in$\mathrm{lab}$ and outdoor experiments. The former indicate that the Atheros AR5213A chipset based interfaces they equipped were indeed compliant with the spectral mask requirements of the 802.11a specification [5]. Their testbed was again based on a single board Linux-based PC that hosted the two interfaces and used the madWifi driver. They mention no board crosstalk and using omnidirectional antennas for their outdoor testbed they suggest increasing channel separation and antenna distance as well as using directional antennas in order to mitigate the effects of 802.11a ACI, which they report that reduces 
performance because on a multi-interface node the transmitter can interfere with its own receiver on a different channel.

In [8] the authors produce a simple model to theoretically quantify the ACI power leakage. Their key idea is focused on taking an integral over the whole overlapping region of the interfering channels spectral masks. Due to the simplicity of their model they apply it to the spectral masks of $802.11 \mathrm{~b} / \mathrm{g}$ and that of 802.16. They state that the use of partially overlapped channels is not always harmful. Furthermore they state that a careful use of some partially overlapped channels can often lead to significant improvements in spectrum utilization and application performance, with respect to the interfering nodes' distances.

In our previous work [9] we utilized the model for calculation of the interference power by partially overlapping channels introduced in [8] and combined it with the Signal to Interference plus Noise (SINR) criterion for signal capture to quantify the effect of ACI in 802.11a. We validated the results from our theoretical model by applying it on an in-lab testbed, in which we use signal splitters/combiners and fixed attenuators to emulate the wireless channel [10]. Our experimental setup was able to isolate the mechanisms that the neighboring channel interference affects the 802.11a: the packet capture at the receiver and the Clear Channel Assessment (CCA) mechanism. Through experimentation we quantified the effect of ACI for both of the mechanisms above. In order to avoid any performance issues related to hardwareWLAN interface or operating system-madWifi interaction, we had used a single laptop for each radio interface, a practice that we employed in this work in the same fashion as $[11,12]$. Results indicated that using multiple 802.11a interfaces in a single location requires careful channel allocation and physical antenna separation, since throughput can be severely degraded, due to radio issues alone.

The authors of [12] conducted a measurement study to examine the performance and configuration of a multi-radio $802.11 \mathrm{~g}$ node. Their findings verified that the placement (orientation and distance) of directional antennas on a multihop node have significant impact on the achieved throughput. Part of the observed behavior was attributed to the beam patterns of the directional antennas used in the measurements. This is to our knowledge the only work that attempts to quantify and explore the usage of directional antennas in a systematic fashion.

\section{Paper Overview \& Organization}

In this work we expand the model we introduced in $[9,10]$. This is done so that the ACI calculation can take into account the large-scale path loss, radiation parameters of the antennas equipped and their relative location. We conducted thorough and systematic outdoor experiments of medium-range links that quantify the effect on achieved throughput of patch panel directional antennas in an 802.11a multi-radio testbed.

Our key result is that we identified the points of failure in the theoretical link budget and throughput prediction model that were not evident on a wired emulation testbed: Firstly the coupling with the path loss model can introduce errors when path loss is calculated for short distances. Secondly parameters reported by hardware vendors and drivers may not always be the actual ones and therefore some calibrating measurements are needed in order verify/rectify them. Finally, collocated 802.11a nearby channel nodes are affected symmetrically. All perceive the rest as "interferers" as they all comply with the same 802.11a medium access scheme and have similar physical layer requirements. This also implies that interference caused by $802.11 \mathrm{a}$ interfaces is structured and cannot be considered as AWGN. Therefore further analysis and model refinement is required to more accurately capture these interactions.

Our next steps are summarized in two actions: having identified the parameters in need of rectification we aim to use our model to produce a tool that, including a minimal systematic calibration procedure, will be used for the design and configuration of a multi-radio multi-hop mesh network that uses off-the-shelf 802.11a interfaces and antennas. Also, we aim to launch a large scale measurement campaign for a variety of environments in order to identify the effect of environmental parameters, omitted in this as in previous works.

The rest of the paper is organized as follows: section II describes our theoretical model and based on that introduces a link budget calculation that can be used for goodput prediction. Section III describes the testbed we used and the experiments we conducted along with their design rationale. The results obtained by the experiments are presented in section IV. Our conclusions from this work and our plans for future works are detailed in section $\mathrm{V}$.

\section{Modeling ACI For Link Budget AND ThroughPUT CALCULATIONS}

\section{A. ACI Modeling}

As we thoroughly presented in [10], the ACI power at a node $i$ can be calculated as a sum of the interfering powers that to that node

$$
I_{i}=\sum_{j \neq i} P_{j} \cdot \xi_{j, i} \cdot G_{j, i}
$$

where $P_{j}$ is the transmitting power of node $j$ in watts, $G_{i, j}$ the path loss of from $j$ to $i$ and the factor $\xi_{j, i}$ depends on the spectral properties (inter-channel spectral distance, channel width and spectral mask) of the channels and receivers used and the radio channel separation between interferer $j$ and receiver $i$. Apparently, if links $i$ and $j$ use the same channel then we are reduced to a co-channel case and so $\xi_{j, i}=1$. We calculate the $\xi$ factor normalizing the spectral mask $S(f)$ within a bound $w$ that can be as narrow as the nominal channel width and then filter this normalized $S^{\prime}(f)$ over the frequencies that will be within the band-bass filter of the receiver. Ideally this should be a flat band-pass $20 \mathrm{MHz}$ filter, but in the typical case the assumption authors of [8] holds, and so in the general case we have:

$$
S^{\prime}(f)=\frac{S(f)}{\int_{-\frac{w}{2}}^{\frac{w}{2}} S(f) d f}
$$


where $w$ is the receiver filter bandwidth, and so:

$$
\xi_{i, j}=\int_{-\frac{w}{2}}^{\frac{w}{2}} S^{\prime}(f) \cdot S^{\prime}\left(f-f_{\text {int }}\right) d f
$$

Using our model and the spectral mask for the 802.11a mandated by the standard in [5] we analytically calculated the theoretical power leakage from two neighboring 802.11a channels. Table I holds the ACI values for a single interfering node assuming a $G_{i j}$ of 1 - effectively this is the $\xi_{j, i}$ factor expressed in $\mathrm{dB}$.

\section{B. Link Budget Caclulation}

Using eq. 1 above and the calculated values of $\xi_{i, j}$ we can perform cross-channel link budgets for use in the Signal-toInterference-plus-Noise (SINR) model. To do this we assume that ACI can be considered as white Gaussian noise. Doing this the SINR can then be calculated at any receiver $i$ as in eq.4 below:

$$
\operatorname{SINR}_{i}=\frac{P_{i} \cdot G_{i, i}}{N_{i}+I_{i}}
$$

Here $P_{i}$ is the transmitter power of link $i$, and $G_{i, i}$ denotes the path loss along link $i . N_{i}$ is the noise power at receiver. As in eq.1 all powers are expressed in watts and $G_{i, i}$ is a dimensionless scaling factor.

Each path loss in eq. 1 and eq. 4 consists of the long-scale path loss plus the loss from any connectors/cables plus the antenna gains at the incident angles of the link. In eq. 5 we have the calculation of $G_{i, j}$ in $\mathrm{dB}$; all other losses and gains are likewise in $\mathrm{dB}$ :

$$
G_{i, j}(d B)=P L_{i, j}+C L_{i}+C L_{j}+A G_{i}\left(\theta_{i, j}\right)+A G_{j}\left(\theta_{j, i}\right)
$$

The path loss $P L_{i, j}$ can be typically calculated from well known models [14] for links longer than the near field of the antennas used. The $C L_{i}, C L_{j}$ losses can be calculated by looking up the nominal loss values provided by the extension cables, pigtails, etc. used to connect the wireless interface to the antenna. The antenna gain at the incident angle $\theta_{i, j}$ can be calculated from the radiation pattern, subtracting the gain degradation at the bearing of $\theta_{i, j}$ from the nominal antenna gain (for the trivial case of $\theta_{i, j}=0$ then $A G_{i}$ equals the nominal antenna gain).

\section{Expected Throughput}

In [15] the authors derive an analytical model that couples SNR with the maximum effective throughput (goodput) of an 802.11a link. Assuming that interference is equivalent to AWGN we could couple the SINR calculation from eq.4 with eq.3 and eq.7 [16] and thus predict the goodput of any link under ACI. As we already know from our previous work [10], ACI created with 802.11a interfaces does not have the properties of AWGN since the interfering link adheres to the 802.11a wireless medium access scheme (the 802.11a Distributed Coordination Function - DCF). Therefore in the following experiments we were indeed expecting that the model so far described would underestimate the measured throughput.

\section{TESTBED AND EXPERIMENTS' SETUP}

We conducted our experiments on a testbed platform of 4 laptops running on Linux with the 2.6.20-16 kernel. We chose to emulate a dual-radio node by using two laptops, one for each WLAN interface, to avoid altogether any possible performance issues related to hardware-WLAN interface or operating system-MadWifi interaction. The wireless interfaces we used were 4 Atheros-based Ubiquiti SRC $802.11 \mathrm{a} / \mathrm{b} / \mathrm{g}$ pcmcia cards running on the MadWifi-ng driver (svn 2594). We used a Rohde\&Schwarz FSH6 Spectrum Analyzer in order to verify the output power and spectral masks produced by the wireless interfaces. We also used a laptop with the Airmagnet Laptop Analyzer (v.4), to have a sniffer's view of the actual 802.11a MAC layer and verify received signal power. For UDP traffic generation we used the Iperf program (v2.0.2).

The antennas equipped were Interline's $19 \mathrm{dBi}$ patch panels and Vesuvius Streamline's $27 \mathrm{dBi}$ patch panels. We also used mmcx-to-N pigtails and $5 \mathrm{~m}$ long N-to-N terminated LMR-400 extension cables to physically separate the antennas. The antennas were mounted on tripods on one end of the links and on a $1.7 \mathrm{~m}$ tall $2.5 \mathrm{~m}$ wide post on the other. Figure 1 is an onsite photo of the post, with two of the $27 \mathrm{dBi}$ antennas mounted on it; Figure 2 is a photo of the tripods with the $19 \mathrm{dBi}$ antenna panels during the initial calibration measurements.

According to the specifications of the antennas the front-toback ratio is at least $25 \mathrm{~dB}$ in the $19 \mathrm{dBi}$ patches and at least 35 $\mathrm{dB}$ in the $27 \mathrm{dBi}$ case. Radiation patterns are provided by the vendor (see figure 3). With those, the model of the previous section assumes that $A G\left(\theta_{i, j}\right)$ in eq.5 can be calculated.

During the early experiments, we found a great diversion in the results from our predictions. This led us to scrutinize both the values provided by the vendors of the hardware and the models for calculation of the path loss for short distances, and so introduce some calibration measurements to verify the output power of the transmitters, the path loss and the antenna pattern.

The goal of our experiments was to examine the applicability of the model presented in section 2, at the near end of a multi-radio node with directional antennas in outdoors environments, since we had already verified its applicability on the in-lab wireless emulation testbed, where cables and attenuators emulated the wireless medium.

The wireless testbed was set up on the roofs of two buildings $250 \mathrm{~m}$ apart in the campus of FORTH, at a height of $10 \mathrm{~m}$ with a Fresnel zone clearance of approximately $8 \mathrm{~m}$.

Two parallel links were established between the two buildings. The antenna separation at the ends of the links was either $1.5 \mathrm{~m}$ or $3 \mathrm{~m}$. The antennas used were either the $19 \mathrm{dBi}$ panels on all nodes or the $27 \mathrm{dBi}$ panels on all nodes. The transmit powers used were either $1 \mathrm{dBm}$ or $10 \mathrm{dBm}$. Diversity was disabled by the MadWifi driver and the basic rate was locked at $6 \mathrm{Mbps}$. 
TABLE I. ACI FACTOR $\xi_{i, j}$ LIMITS (IN dB) FOR 1 CHANNEL AND 2 CHANNELS AWAY

\begin{tabular}{|c|c|c|}
\hline $\begin{array}{c}\text { Receiver } \\
\text { Bandwidth } \\
w\end{array}$ & $\begin{array}{c}\text { Adjacent } \\
\text { Channel Power } \\
\text { Leakage }\end{array}$ & $\begin{array}{c}\text { Next Adjacent } \\
\text { Channel Power } \\
\text { Leakage }\end{array}$ \\
\hline $20 \mathrm{MHz}$ & -22.04 & -39.67 \\
\hline$\infty$ & -19.05 & -36.67 \\
\hline
\end{tabular}

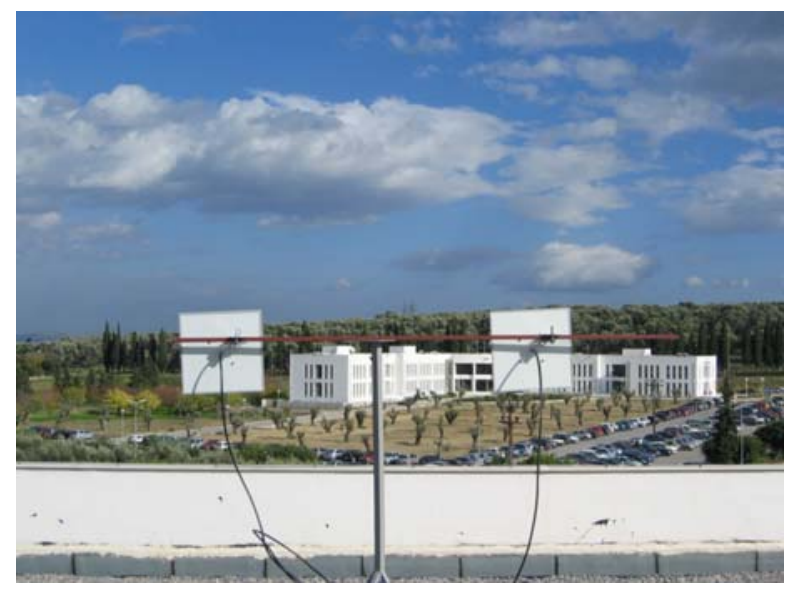

Figure 1. The $27 \mathrm{dBi}$ patch pannels at the roof of FORTH on the mounting pole used.

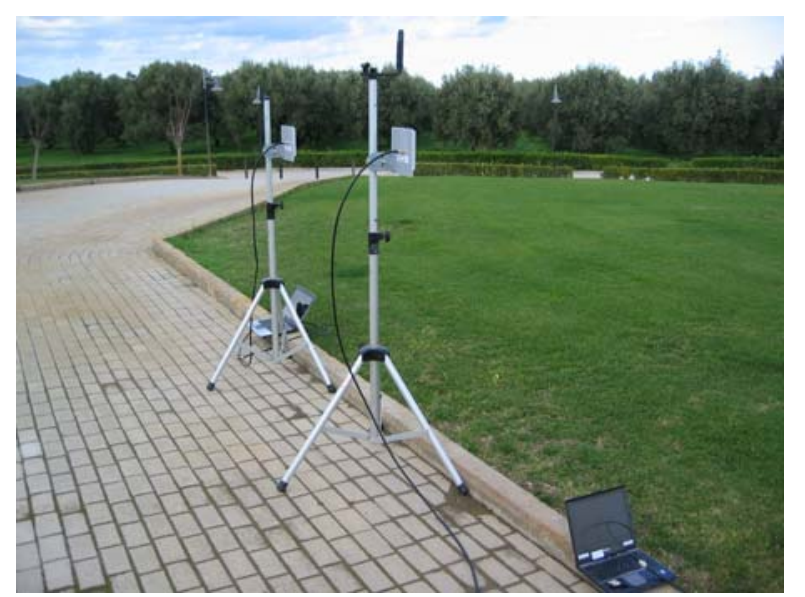

Figure 2. The 19dBi patch pannels mounted on the tripods during some of the calibration measurements
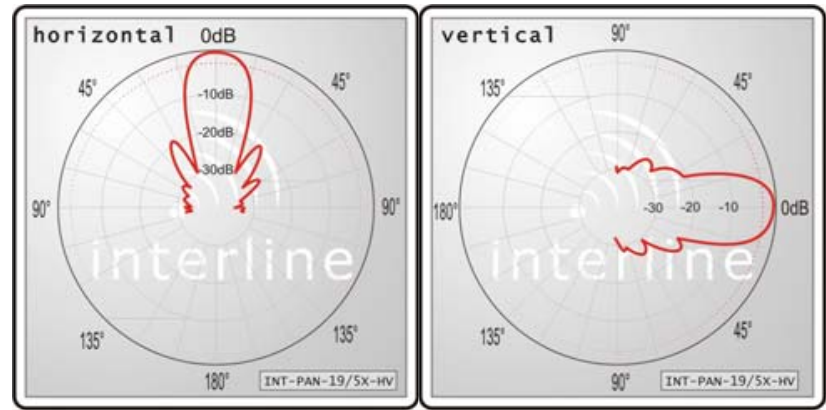

Figure 3. The radiation patterns for the $19 \mathrm{dBi}$ antennas used.
With these parameters we tested how one of the links would perform in terms of throughput, under interference generated mainly on one of the end nodes, from either the adjacent 802.11a channel or from the next adjacent channel; typically we would run the test on a link in channel 60 and the interferer would be in channel 56 or 52 . The interfering link was in all cases fixed at the rate of $6 \mathrm{Mbps}$, running UDP traffic with full payload packets, thus producing the maximum possible in 802.11 a channel utilization of approximately $90 \%$. It is also obvious that the "interfering" link suffered the same ACI effects from the test link leakage.

The test process was the following: the test link would run a 30sec UDP flow for each rate supported by the 802.11a, for 3 payload sizes: 500, 1000 and 1470 Bytes, under the influence of the interferer. For stability and reproducibility purposes we would restart the MadWifi driver with the new rate parameter between each run. To this end the entire process was shellscripted and so each test of the link resulted in a runtime of approximately 40 minutes due to synchronization and loading/unloading overheads. In all cases a single baseline run was conducted without the presence of the interferer.

The path between the two buildings is above the parking lot of FORTH. The majority of the experiments were conducted during weekends and therefore the lot was practically empty. For validation purposes we performed a few tests with a full parking lot.

The tests were designed to examine how an interface will be affected by another interface of the same multi-radio node equipped with directional antennas due to ACI. In [10] we had identified the two mechanisms that are affected due to radio ACI: (i) The packet capture and (ii) the Clear Channel Assessment of 802.11a. The first is a PHY mechanism and reduces goodput by increasing the packet error rate at the receiver, while the second is a MAC mechanism that results in a transmitter falsely deferring during the DCF and thus again goodput reduction. Two major classes of tests were performed in order to look into the two mechanisms: The first was conducted with the receiver of the test link in the same locale with the transmitter of the interfering link, while in the second the transmitters of both links were collocated. Link budget calculations had been performed to ensure that only the nearend ACI exists.

\section{RESULTS}

Conducting the early tests of the experiment we noticed that our model was not able to estimate the results. Narrowing down the fault factors we tested the input values for the path loss model described in section 2. For this we used the Airmagnet Laptop analyzer, to get the per packet received signal power at the testbed we had set up. We noticed that the measured value would significantly vary from the estimated.

This misestimation was attributed to three possible sources: First the output power of the wireless interfaces was actually not the one reported by the MadWifi driver. Specifically setting the txpower option to $10 \mathrm{dBm}$, would make the card transmit at a power of approximately $15 \mathrm{dBm}$, while $1 \mathrm{dBm}$ at the driver, would produce an output power of about $12 \mathrm{dBm}$. These values were obtained with our spectrum analyzer. Second, we had 
calculated the physical separation of the antennas to be in their far field and so we expected the radiation patterns (e.g. of figure 3) provided by the vendors to hold. Taking the per packet received signal power we found that either the antenna radiation pattern or that the far field calculation [14] was inaccurate. In such a case neither the path loss Friis equation, nor the radiation pattern should have been used. Finally, the ACI produced by an 802.11a interface is not a signal of constant power over time. Hence the assumption that it can be approximated as AWGN is very harsh for the calculated SNR.

As mentioned before, we performed two classes of experiments in order to look into the two mechanisms that are affected by ACI. The first was conducted with the receiver of the test link in the same locale with the transmitter of the interfering link, while in the second the transmitters of both links were collocated. In order to clearly separate the two mechanisms the link budget calculations in the results presented were based on the measurements, rather than the initial model.

Also we have to note that as mentioned before we conducted a series of experiments during a weekday with the parking lot full of cars. These experiments were reproduced during a weekend session and the results varied within the $95 \%$ confidence interval of the measurements. This was expected due to the $8 \mathrm{~m}$ Fresnel zone clearance ${ }^{1}$. Still the presented figures do not contain any data from that session, for completeness reasons.

In all figures we present mean values obtained over the runtime of the tests. We have a baseline run for a single link at channel 60. In figure 4 the antennas attached to the nodes were the $19 \mathrm{dBi}$ panels and the txpower was set to $10 \mathrm{dBm}$, producing a $15 \mathrm{dBm}$ output, as mentioned above. As we can see an antenna separation by $3 \mathrm{~m}$ is sufficiently close to the baseline when the transmit power of the interfering link is high even when the channel distance is only one. On the other hand $1.5 \mathrm{~m}$ is not sufficient separation for the ACI to fade over this path, so it reduces the SNR at the receiver increasing the PER and thus reducing throughput. Reducing the transmission power to $1 \mathrm{dBm}$ nominally (a real reduction of $3 \mathrm{~dB}$ ) results in very small PER.

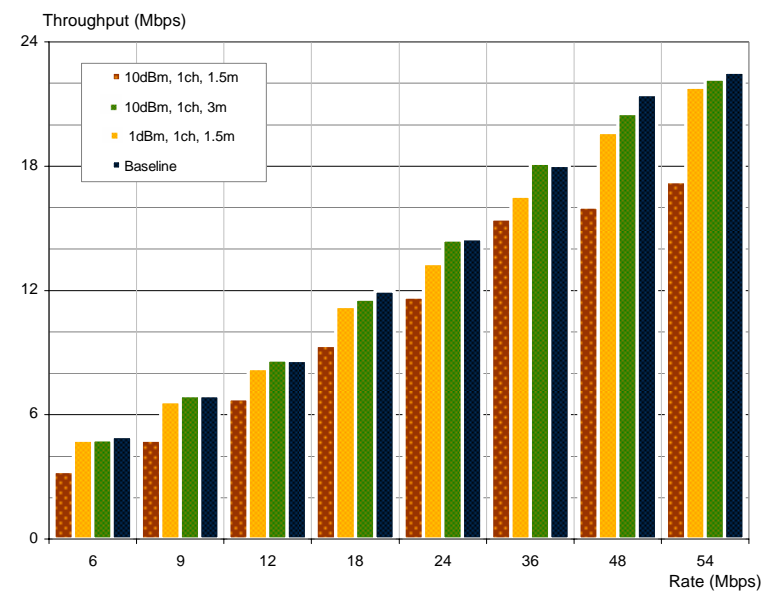

Figure 4. Physical distance of interfering antennas and power effects on the the packet capture mechanism for the antennas of $19 \mathrm{dBi}$ gain.
In figure 5, we show that using a second interface 2 channels apart produces no significant ACI as the perceived throughput practically matches the baseline case. Using higher gain antennas results in overcoming the ACI effect due to better beamforming that allows less power to leak in angles outside the main lobe. This can be clearly seen comparing the $19 \mathrm{dBi}$ to $27 \mathrm{dBi}$ bars.

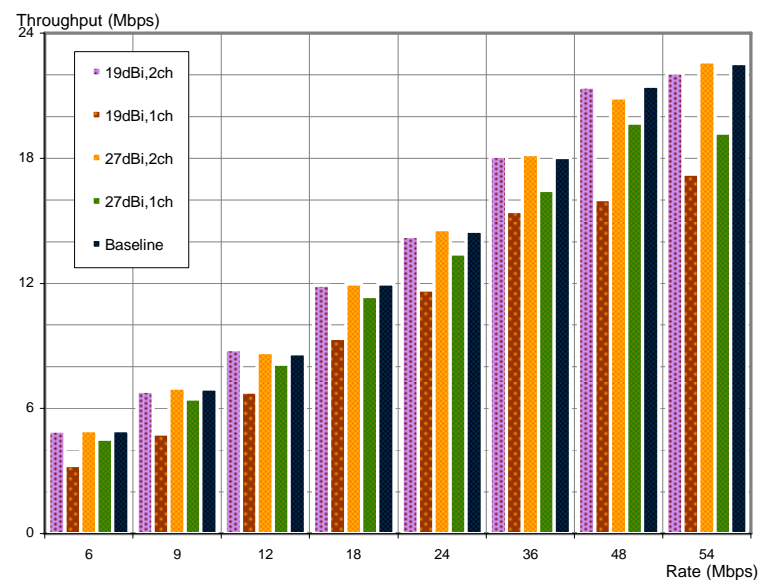

Figure 5. Distance of interfering channel and antenna gain effects on the the packet capture mechanism, for txpower of $10 \mathrm{dBm}$ and antennas at $3 \mathrm{~m}$ apart. Payload in the UDP flow of the test link is set to 1000Bytes.

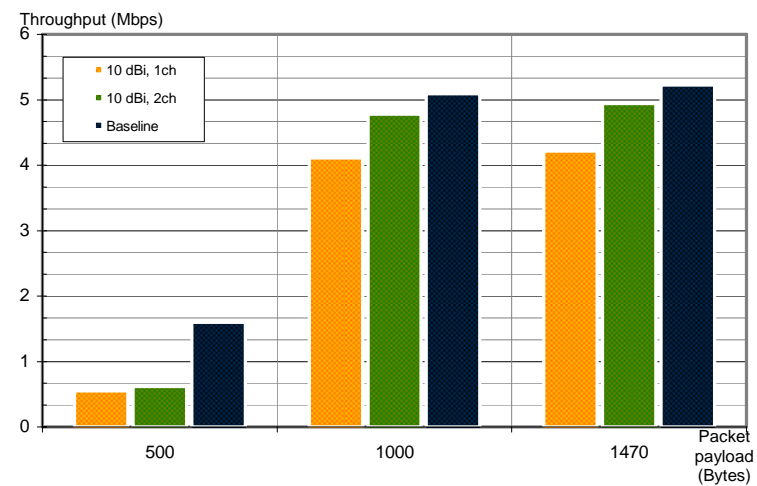

Figure 6. Effect of ACI on the CCA mechanism for the 6Mbps rate with $19 \mathrm{dBi}$ antennas separated by $3 \mathrm{~m}$.

Figure 6, illustrates the effect of ACI on the CCA mechanism for three different packet sizes which correspond to three different channel utilization values. We see that in the low utilization scenario the CCA mechanism is affected the most and regardless of the channel separation. Higher utilizations are more robust and this can be attributed to the symmetrical nature of the interference produced in the testbed (interferer's throughput/utilization is also affected).

\section{CONCLUSIONS \& FUTURE WORK}

We presented an outdoors 802.11a testbed based on off-the shelf components that we used for multi-radio mesh node experimentation. This is the first such testbed, to our knowledge, equipped with directional antennas. With it we conducted a thorough and systematic set of measurements, in medium range outdoors links, to examine how the physical location of the antennas, the output power of the interfaces and

\footnotetext{
The first Fresnel zone for $250 \mathrm{~m}$ at $5.3 \mathrm{GHz}$ (channel 60 ) is $2 \mathrm{~m}$.
} 
the channel separation of the links affect the observed throughput. For the setup of the testbed and to estimate the induced ACI we utilized a theoretical model, already verified on an in-lab wireless testbed emulator [10].

Our key result is that we identified points of failure in the theoretical link budget and throughput prediction model that were not evident on a wired emulation testbed in which the path was fully controlled and not subject to the wireless medium.

First coupling with a path loss model can introduce errors when path loss is calculated for short distances. Secondly parameters reported by hardware vendors and drivers (transmit power, antenna patterns) may not always be the actual ones. In order to alleviate these problems calibrating measurements are needed. Both these problems introduce errors in the estimation of the model we had developed that were not observable in the initial paper and still do not reduce the validity of the model: In order to operate the real values need to be inserted rather than values provided by other models.

Another issue is that, collocated 802.11a nearby channel nodes are affected symmetrically. All perceive the rest as "interferers" as they all comply to the same 802.11a medium access scheme and have similar physical layer requirements. This also implies that interference cause by $802.11 \mathrm{a}$ interfaces is structured and cannot be considered as AWGN. Therefore further analysis and model refinement is required to more accurately capture these interactions.

Still, with experience on conducting such experiments and the additional experience of building a city-wide 802.11a multi-radio mesh testbed [13] with links of a few kilometers, we continue to support with confidence the need for such theoretical models that must be aided by few key measurements and calibrations. In this way with minimal measuring overhead such models can save time and effort and can also give significant insight to the design and dimensioning of larger and more complex mesh networks. Such models can readily be extended to other wireless networking technologies as they are based on fundamental principles of wireless communications and their components' parameters are technology dependent.

Our next steps are the following: Having identified the parameters in need of rectification we aim to use our model to produce a tool that including a minimal, calibration procedure will be used for the design and configuration of a multi-radio, multi-hop mesh network that uses off-the-shelf $802.11 \mathrm{a} / \mathrm{b} / \mathrm{g}$ interfaces and antennas. To this end we have to devise the proper calibrating procedure mentioned above and also to refine the model with respect to the fact that 802.11-produced ACI is not correctly modeled as AWGN.

Also, in parallel to this effort, we aim to launch a large scale measurement campaign for a variety of environments in order to identify the effect of environmental parameters such as weather conditions, terrain and population/mobility, as well as other parameters as antenna polarization and vertical spacing, omitted in this as in previous works.

\section{ACKNOWLEDGMENTS}

The authors acknowledge the substantial aid of $\mathrm{Mr}$. Chariton Melissaris during the rooftop experiments and of $\mathrm{Mr}$. Spyros Lyberis with the scripting of the tests.

\section{REFERENCES}

[1] I.F. Akyildiz, X. Wang, W. Wang, "Wireless mesh networks: a survey" Computer Networks, Volume 47 Issue 4, 15 March 2005, p.445-487.

[2] R. Bruno, M. Conti, E. Gregori, "Mesh networks: commodity multihop ad hoc networks", IEEE Communications Magazine, Volume 43, Issue 3, March 2005, p.123-131

[3] P. Gupta and P. R. Kumar. The Capacity of Wireless Networks. IEEE Transactions on Information Theory, 46(2):388-404, March 2000

[4] IEEE 802.11, Wireless LAN Medium Access Control (MAC) and Physical Layer (PHY) Specifications, Standard, IEEE, August 1999.

[5] IEEE 802.11a, Part 11: Wireless LAN, Medium Access Control (MAC) and Physical Layer (PHY) Specifications: High-Speed Physical Layer in the $5 \mathrm{GHz}$ Band, supplement to IEEE 802.11 Standard, September 1999.

[6] J. Robinson, K. Papagiannaki, C. Diot, X. Guo, and L. Krishnamurthy, "Experimenting with a Multi-Radio Mesh Networking Testbed", 1st workshop on Wireless Network Measurements (WiNMee 2005), Italy, Apr. 2005.

[7] C. M. Cheng, P. H. Hsiao, H. T. Kung, and D. Vlah, "Adjacent Channel Interference in Dual-radio 802.11 Nodes and Its Impact on Multi-hop Networking", IEEE GLOBECOM 2006, San Francisco, CA, USA, Nov. 2006.

[8] A. Mishra, et. al. , "Partially Overlapped Channels Not Considered Harmful”, SIGMetrics/Performance'06, Saint Malo, France, Jun., 2006.

[9] V. Angelakis, A. Traganitis, V. Siris, "Adjacent channel interference in a multi-radio wireless mesh node with $802.11 \mathrm{a} / \mathrm{g}$ Interfaces", IEEE INFOCOM 2007, Anchorage, Alaska, USA, May 2007.

[10] V. Angelakis, S. Papadakis, V. Siris and A. Traganitis, "Adjacent Channel Interference in 802.11a: Modeling and Testbed validation", 2008 IEEE Radio and Wireless Symposium, Orlando, FL, USA, Jan. 2008.

[11] F. Potorti, P. Barsocchi and G. Oligeri "Frame error model in rural WiFi networks", WiNMee 2007, Limassol, Cyprus, Apr. 2007.

[12] Michael Zink, Timothy Ireland, Adam Nyzio, James F. Kurose, "The Impact of Directional Antenna Orientation, Spacing, and Channel Separation on Long-distance Multi-hop 802.11g Networks: A Measurement Study", WiNMee 2007, Limassol, Cyprus, Apr. 2007.

[13] V.Angelakis, V. Siris, et. al. "Heraklion MESH: An Experimental Metropolitan Multi-Radio Mesh Network" WiNTECH'07, Montréal, Québec, Canada, Sept., 2007.

[14] Theodore S. Rappaport, Wireless Communications: Principles \& Practice, Prentice Hall, 2nd Edition, 2002.

[15] Daji Qiao, Sunghyun Choi, and Kang G. Shin, "Goodput Analysis and Link Adaptation for IEEE 802.11a Wireless LANs", IEEE Trans. on Mobile Computing, v.1, Nr.4, Oct-Dec 2002. p.p. 278-292.

[16] Jun J., Peddabachagari P., Sichitiu M. "Theoretical Maximum Throughput of IEEE 802.11 and its Applications", IEEE NCA'03, Cambridge MA., USA, Apr. 2003.

[17] Jian Liu , Dongfeng Yuan, Song Ci and Yingji Zhong, "Nonlinear optimization for energy efficiency in IEEE 802.11a wireless LANs", Computer Communications, Volume 29, Issue 17, 8 November 2006, p.p. 3455-3466.

[18] Jung Jee and Hossain Pezeshki Esfahani. "Understaning wireless LAN Performance Trade-Offs". Communications Systems Design Nov. 2002. Online at: http://img. cmpnet.com /commsdesign/csd/ 2002/nov02/feat3nov02.pdf

[19] Burton M.. "Channel Overlap Calculations for 802.11b Networks", Cirond Technologies Inc. 2002. 